\title{
Polyazimuthal Map Projections
}

\author{
Krisztián KERKOVITS
}

Department of Cartography and Geoinformatics, ELTE Eötvös Loránd University, Budapest, Hungary

kerkovits@map.elte.hu

Abstract: A polyazimuthal map projection is a mapping that represents parallels as non-concentric full circles on the plane. Polyazimuthal mappings are almost never mentioned in the literature dealing with map projections. However, these projections are flexible; their distortion characteristics are highly mutable. This paper expands the theory of polyazimuthal map projections. Furthermore, this study also shows the derivation for variants of this projection family (e. g. equal-area, orthogonal). The article concludes with some practical applications in the field of low-distortion map projections to demonstrate their advantages.

Keywords: Polyazimuthal projections, optimal map projections, numerical optimization

\section{Motivation}

What is a polyazimuthal map projection? Just a simple literature search shows that even the terminology is inconsistent among researchers. It is obvious that pseudoconic projections map parallels to concentric circular arcs and polyconic projections map them to non-concentric circular arcs (Frančula and Lapaine 2018). The opinion of the author is that consequently pseudoazimuthal and polyazimuthal projections should be alike: as the former maps parallels to concentric full circles (Bugajevskij and Snyder 1995, p. 129), the latter should map them to non-concentric full circles. This definition can be found in Russian literature (Serapinas 2005, p. 63).

On the other hand, English literature uses the term polyazimuthal projection for different types of map projections. The term may refer to projections that map parallels to concentric ellipses. It may mean projections that map meridians to straight lines, and are conformal at the pole, but the images of the parallels are not circles (Canters 2002, p. 31). Fuller (1975) also calls his polyhedral projection as polyazimuthal. Furthermore, even an animated perspective azimuthal projection with a moving plane is referred to as polyazimuthal (Saalfeld 1997).

The main motivation of this study was a single paragraph in Serapinas (2005, p. 63): "Polyazimuthal map projections can have arbitrary distortion characteristics. Unfortunately, the practical value of these projections is not perfectly clear." The aim of this study is to examine the usefulness of this exotic but flexible class of map projections and discover their properties. The paper provides exact formulae and derivation of low-distortion polyazimuthal map projections and demonstrates that maps using such projections can also have high aesthetic values.

The only considerable work on this topic is due to Tolstova (1981) who reviewed various types of polyazimuthal projections and presented formulae and derivations for certain equal-area and conformal variants, but she did not list general solutions suitable for optimization, she only reported some examples. She was the first to denote this group of mappings as polyazimuthal (полиазимутальная проекиия).

\section{Common Properties}

According to the definition of Serapinas (2005, p. 63), a polyazimuthal map projection is a mapping in which parallels are eccentric circles on the map, meridians appear as curves that meet at the pole, and the centres of the mapped parallel circles lie on the mid-meridian.

The former definition does not mention axial symmetry, but due to aesthetic reasons, it is rational to restrict the scope of the study to map projections that are symmetrical to the vertical axis. This implies that the mid-meridian must be straight on the map. Such polyazimuthal map projections may never be

KiG No. 32, Vol. 18, 2019, https://doi.org/10.32909/kg.18.32.2 - - 


\title{
Poliazimutne kartografske projekcije
}

\author{
Krisztián KERKOVITS \\ Department of Cartography and Geoinformatics, ELTE Eötvös Loránd University, Budimpešta, Mađarska \\ kerkovits@map.elte.hu \\ Članak je predan na engleskom jeziku. Na hrvatski ga je preveo V. Lapaine. \\ The paper was submitted in English. It was translated into Croatian by V. Lapaine.
}

Sažetak: Poliazimutna kartografska projekcija je preslikavanje paralela kao nekoncentričnih kružnica u ravnini projekcije. Poliazimutna preslikavanja se ne spominju gotovo nigdje u literaturi o kartografskim projekcijama. Međutim, te su projekcije fleksibilne, a njihove distorzije jako promjenljive. Ovaj članak proširuje teoriju poliazimutnih projekcija. Nadalje, ova studija također daje izvod različitih varijanti takvih projekcija (npr. ekvivalentne, ortogonalne). Članak završava s nekoliko praktičnih primjena u području kartografskih projekcija s malim distorzijama kako bi se demonstrirale njihove prednosti.

Ključne riječi: poliazimutne projekcije, optimalne projekcije, numerička optimizacija

\section{Motivacija}

Što je to poliazimutna projekcija? Jednostavno pretraživanje literature pokazuje da terminologija među istraživačima nije konzistentna. Očito je da pseudokonusne projekcije preslikavaju paralele u koncentrične kružne lukove, a polikonusne ih preslikavaju na nekoncentrične kružne lukove (Frančula i Lapaine 2018). Prema mišljenju autora pseudoazimutne i poliazimutne trebalo bi definirati na sličan način: prve preslikavaju paralele na koncentrične kružnice (Bugajevskij i Snyder 1995, str. 129), a druge na nekoncentrične kružnice. Takva se definicija može naći u ruskoj literaturi (Serapinas 2005, str. 63).

$S$ druge strane engleska literatura upotrebljava termin poliazimutne projekcije za drukčiji tip projekcija. Taj se naziv može odnositi na projekcije koje preslikavaju paralele na koncentrične elipse. Može značiti i projekcije koje preslikavaju meridijane u pravce, konformne su u polu, no slike paralela nisu kružnice (Canters 2002, str. 31). Fuller (1975) također naziva svoju poliedarsku projekciju poliazimutnom. Nadalje, čak se i animirana perspektivno azimutna projekcija s pomičnom ravninom naziva poliazimutnom (Saalfeld 1997).

Glavna motivacija za ovaj rad bio je jedan odlomak u Serapinasovoj knjizi (2005, str. 63): "Poliazimutne projekcije mogu imati proizvoljne karakteristike distorzija. Nažalost, praktična vrijednost tih projekcija nije sasvim jasna." Cilj je ovoga rada istražiti korisnost te egzotične, ali fleksibilne klase projekcija i otkriti njihova svojstva. Članak daje egzaktne formule i izvode za poliazimutne projekcije s malim distorzijama i pokazuje da karte izrađene $u$ takvim projekcijama mogu imati veliku estetsku vrijednost.

Jedini značajniji rad na tu temu je onaj Tolstove (1981) koji prikazuje različite tipove poliazimutnih projekcija i daje formule i izvode za izvjesne ekvivalentne i konformne varijante, ali ne daje opća rješenja pogodna za optimiranje. Ona daje samo primjere i prva je koja je imenovala tu skupinu projekcija kao poliazimutne (полиазимутальная проекиия).

\section{Svojstva poliazimutnih projekcija}

Prema Serapinasovoj definiciji (2005, str. 63), poliazimutna projekcija je preslikavanje kod kojega su paralele na karti ekscentrične kružnice, meridijani krivulje koje se susreću u polu, a središta kružnica, koje su slike paralela, leže na srednjem meridijanu. Ta definicija ne spominje osnu simetriju, no zbog estetskih razloga razumno je ograničiti istraživanje na projekcije koje su simetrične s obzirom na vertikalnu os. To implicira da slika srednjeg meridijana mora biti pravac. Takve poliazimutne projekcije neće nikad biti simetrične u odnosu na horizontalnu os jer bi u tom slučaju slike paralela bile koncentrične.

Sve su preslikane paralele kružnice pa je, prema tome, slika infinitezimalne kružnice sa središtem $u$ 
symmetrical to the horizontal axis, as this would mean that parallels are concentric.

All mapped parallels are circles; consequently, the image of the infinitesimal circle centred on the pole is a circle on the map. According to the theorem of Tissot (1878), this means that such mappings must be conformal at the pole. This work will often use this result. Due to the previous arguments, it is clear that the normal aspect of a polyazimuthal mapping is a good choice for areas that

- are large enough so that the lower distortion of such complicated projections is visible when compared to traditional, simpler mappings,

- lie on high latitudes where the angular distortion must be low,

- and are highly asymmetrical to the pole.

The notational system of the paper is as follows: If the mapped area is large, the difference between the ellipsoid of revolution and the sphere is negligible; therefore, the reference frame will be a unit sphere. The sphere is parametrized by the colatitude $\delta$ and the longitude $\lambda$ eastwards from an arbitrary midmeridian $\lambda_{m}$. The colatitude $\delta$ is calculated from the latitude $\varphi$ as $\delta=\pi / 2-\varphi$.

The planar coordinates are described by usual Cartesian coordinates $x, y$. To gain simple formulae, polar coordinates are also introduced:

$\varrho(\delta)$ stands for the radius of the mapped parallel circle with colatitude $\delta$ (Figure 1). @ must be a strictly increasing function. It must be zero at the pole to gain a continuous representation. $c(\delta)$ denotes the distance between the axis $x$ and the centre of the mapped parallel circle. The derivative $d c / d \delta$ must be zero at the pole. It is necessary because a $c(\delta)$ function not fulfilling the previous condition would result in linear scales that depend on the longitude at the pole. This would contradict a previous statement, according to that there is conformity at the pole.

The polar angle measured in the centre of the parallels counter-clockwise from the negative axis $y$ is denoted by $\omega(\delta, \lambda)$. The auxiliary angle $\chi=\omega-\lambda$ will also be used, which is an odd function of $\lambda$ due to the prescribed axial symmetry. The straight mid-meridian enforced by the axial symmetry means that

$$
\forall k \in \mathbb{Z}: \chi(\delta, k \pi) \equiv 0 .
$$

To yield a continuously differentiable representation of the anti-meridian, $\chi$ must also fulfil

$$
\forall k \in \mathbb{Z}: \chi(\delta, \lambda+2 k \pi)=\chi(\delta, \lambda) .
$$

These altogether mean that $\chi$ is a periodic, odd function of $\lambda$ with a period of $2 \pi$.

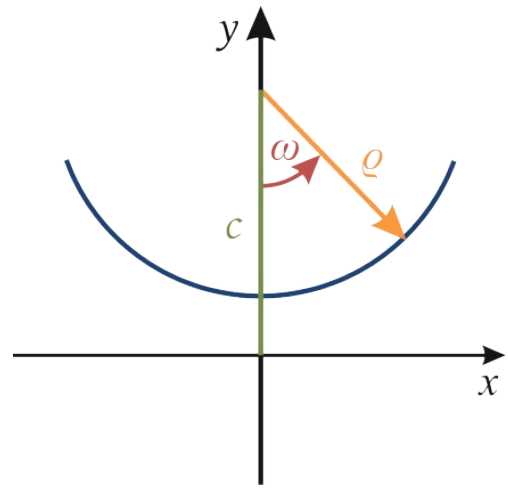

Fig. 1 Polar coordinates used in this paper.

Slika 1. Polarne koordinate upotrijebljene u ovom članku.

Following equations describe the conversion between Cartesian and polar coordinates (cf. Figure 1):

$$
\begin{aligned}
& x=\varrho \sin \omega \\
& y=c-\varrho \cos \omega .
\end{aligned}
$$

Formulae of map projections are usually smooth. (A vector-vector function is called smooth in this study if its partial derivatives of arbitrarily high order are continuous.) However, this smoothness is usually not present at the poles. In spite of this, while seeking the map projection with the lowest distortion possible, the author experienced that distortion isolines could follow the boundary more effectively when smoothness was enforced at the pole. This is desirable according to the theorem of Čebišev (1856). It is reasonable to assume smoothness at the pole: Polyazimuthal mappings are a good choice for regions at high latitudes where the pole will not appear at the edge of the map, rather inside the area of interest. Although there is no rigorous proof, it is likely that optimal map projections are smooth at inner points of the represented area.

This imposes further conditions. Although $\delta$ may never be negative, it is a necessary condition of smoothness at the pole that $\varrho(\delta)$ is odd and $c(\delta)$ is even. This will guarantee that high-order derivatives of linear scales are continuous in all directions.

Tolstova (1981) preferred polyazimuthal projections that have a true-scale mid-meridian. This results in the relation $c=\varrho-\delta$, meaning that Tolstova's $c$ is an odd function. Consequently, her mappings are not smooth at the pole. In this paper, the differentiability of map projections is considered more important than a true-scale mid-meridian; polyazimuthal map projections developed in this paper will not have the latter property.

KiG No. 32, Vol. 18, 2019, https://doi.org/10.32909/kg.18.32.2 - 
polu kružnica na karti. Prema Tissotovom teoremu (1878) to znači da takvo preslikavanje mora biti konformno u polu. U ovom će se radu taj rezultat često upotrebljavati. Zbog prethodnih argumenata, jasno je da je normalni aspekt poliazimutne projekcije pogodan izbor za područja koja:

- su dovoljno velika pa su manje distorzije takvih kompliciranih projekcija vidljive u usporedbi s tradicionalnim, jednostavnijim preslikavanjima

- leže na većim širinama gdje distorzije kutova moraju biti male

- su vrlo asimetrična prema polu.

Sustav oznaka u članku je sljedeći: ako je područje preslikavanja veliko, razlika između rotacijskog elipsoida i sfere je zanemariva, dakle referentna ploha bit će jedinična sfera. Sfera je parametrizirana zenitnom daljinom (colatitude) $\delta$ i geografskom dužinom $\lambda$ istočno od proizvoljnog srednjeg meridijana $\lambda_{m}$. Zenitna se daljina $\delta$ računa iz geografske širine $\varphi$ kao $\delta=\pi / 2-\varphi$.

Koordinate u ravnini opisane su uobičajenim kartezijevim koordinatama $x, y$. Da bi se dobile jednostavne formule, uvode se polarne koordinate:

$\varrho(\delta)$ označava polumjer kružnice koja je slika paralele kojoj odgovara zenitna daljina $\delta$ (slika 1$)$. $\varrho$ mora biti strogo rastuća funkcija. Njezina vrijednost u polu mora biti nula kako bi se dobio prikaz bez prekida. $c(\delta)$ označava udaljenost između osi $x$ i središta kružnice koja je slika paralele. Derivacija $d c / d \delta$ mora biti jednaka nuli u polu. To je nužno jer bi funkcija $c(\delta)$, koja ne bi ispunjavala taj uvjet, dala linearno mjerilo koje ovisi o geografskoj dužini u polu. To bi bilo kontradiktorno prethodnoj tvrdnji prema kojoj je projekcija u polu konformna.

Polarni kut $\mathrm{s}$ vrhom $\mathrm{u}$ središtu slike paralele i mjeren $\mathrm{u}$ suprotnom smjeru od kazaljke na satu od negativnog smjera osi $y$ označen je $s \omega(\delta, \lambda)$. Pomoćni kut $\chi=\omega-\lambda$ treba biti neparna funkcija od $\lambda$ zbog pretpostavljene osne simetrije. Pravocrtni srednji meridijan zbog zadane osne simetrije implicira

$\forall k \in \mathbb{Z}: \chi(\delta, k \pi) \equiv 0$.

Da bi se dobio neprekidan diferencijabilan prikaz antimeridijana, $\chi$ mora ispuniti sljedeće

$$
\forall k \in \mathbb{Z}: \chi(\delta, \lambda+2 k \pi)=\chi(\delta, \lambda)
$$

To znači da je $\chi$ periodična, neparna funkcija $\lambda \mathrm{s}$ periodom $2 \pi$.

Sljedeće jednadžbe opisuju konverziju između kartezijevih i polarnih koordinata (vidi sliku 1):

$$
\begin{aligned}
& x=\varrho \sin \omega \\
& y=c-\varrho \cos \omega .
\end{aligned}
$$

Formule su kartografskih projekcija obično glatke. (U ovom se radu funkcija naziva glatkom ako su njezine parcijalne derivacije bilo kojeg reda neprekidne.) Međutim, ta glatkoća često ne postoji u polovima i autor je ustanovio da, kad se traži projekcija s najmanjom mogućom distorzijom, izolinije distorzije mogu učinkovitije slijediti rub područja ako je postavljen uvjet glatkoće $u$ polu. To je poželjno $u$ skladu s teoremom Čebiševa (1856). Ima smisla pretpostaviti glatkoću u polu - poliazimutna preslikavanja su dobar izbor za područja u većim širinama kod kojih pol neće biti na rubu karte, nego unutar područja. Iako ne postoji strogi dokaz, čini se da su optimalne projekcije glatke $u$ unutrašnjim točkama prikazanog područja.

To postavlja daljnje uvjete. Iako $\delta$ ne može nikada biti negativan, nužan uvjet glatkoće u polu je da je $\varrho(\delta)$ neparna funkcija, a $c(\delta)$ parna funkcija. To će garantirati da su derivacije viših redova linearnog mjerila neprekidne u svim smjerovima.

Tolstova (1981) je preferirala one poliazimutne projekcije koje imaju srednji meridijan bez distorzija. To rezultira relacijom $\mathrm{c}=\varrho-\delta$, što znači da je kod Tolstove $c$ neparna funkcija. Prema tome, njezina preslikavanja nisu glatka u polu. U ovom se radu smatra da je diferencijabilnost projekcije važnija od srednjeg meridijana bez distorzije. Poliazimutne projekcije $u$ ovom radu neće imati to posljednje navedeno svojstvo.

\section{Procjena i optimiranje distorzija poliazimutnih projekcija}

Tradicionalni način procjene distorzija neke projekcije temelji se na Tissotovoj indikatrisi. Najprije treba izračunati kut $\vartheta$ između meridijana i paralela $u$ projekciji (Snyder 1987, Györffy 2002):

$$
\cot \vartheta=\frac{\frac{\partial x}{\partial \lambda} \frac{\partial x}{\partial \varphi}+\frac{\partial y}{\partial \lambda} \frac{\partial y}{\partial \varphi}}{\frac{\partial x}{\partial \lambda} \frac{\partial y}{\partial \varphi}-\frac{\partial y}{\partial \lambda} \frac{\partial x}{\partial \varphi}}=\frac{\frac{d c}{d \delta} \sin \omega+\varrho \frac{\partial \omega}{\partial \delta}}{\frac{d c}{d \delta} \cos \omega-\frac{\partial \varrho}{\partial \delta}} .
$$

Zatim se izračunaju linearna mjerila $u$ smjerovima meridijana i paralela:

$$
h=\sqrt{\left(\frac{\partial x}{\partial \varphi}\right)^{2}+\left(\frac{\partial y}{\partial \varphi}\right)^{2}}=\frac{\frac{d \varrho}{d \delta}-\frac{d c}{d \delta} \cos \omega}{\sin \vartheta}
$$




\section{Estimation and Optimization of Distortions in Polyazimuthal Map Projections}

The traditional way to estimate map distortion is based on the Tissot indicatrix. As a first step, one should calculate the mapped angle $\vartheta$ between the meridians and parallels (Snyder 1987, Györffy 2002):

$$
\cot \vartheta=\frac{\frac{\partial x}{\partial \lambda} \frac{\partial x}{\partial \varphi}+\frac{\partial y}{\partial \lambda} \frac{\partial y}{\partial \varphi}}{\frac{\partial x}{\partial \lambda} \frac{\partial y}{\partial \varphi}-\frac{\partial y}{\partial \lambda} \frac{\partial x}{\partial \varphi}}=\frac{\frac{d c}{d \delta} \sin \omega+\varrho \frac{\partial \omega}{\partial \delta}}{\frac{d c}{d \delta} \cos \omega-\frac{\partial \varrho}{\partial \delta}} .
$$

Next, the linear scales are calculated in the directions of meridians and parallels:

$$
\begin{aligned}
& h=\sqrt{\left(\frac{\partial x}{\partial \varphi}\right)^{2}+\left(\frac{\partial y}{\partial \varphi}\right)^{2}}=\frac{\frac{d \varrho}{d \delta}-\frac{d c}{d \delta} \cos \omega}{\sin \vartheta} \\
& k=\frac{\sqrt{\left(\frac{\partial x}{\partial \lambda}\right)^{2}+\left(\frac{\partial y}{\partial \lambda}\right)^{2}}}{\cos \varphi}=\frac{\varrho \frac{\partial \omega}{\partial \lambda}}{\sin \delta} .
\end{aligned}
$$

Finally, the semi-axes $a$ and $b$ of the Tissot indicatrix are:

$$
\begin{aligned}
& a=\frac{\sqrt{h^{2}+k^{2}+2 h k \sin \vartheta}+\sqrt{h^{2}+k^{2}-2 h k \sin \vartheta}}{2} \\
& b=\frac{\sqrt{h^{2}+k^{2}+2 h k \sin \vartheta}-\sqrt{h^{2}+k^{2}-2 h k \sin \vartheta}}{2} .
\end{aligned}
$$

The overall distortion value containing both the effects of the areal and angular distortions over large areas $S$ can be measured effectively using the AiryKavrajskij criterion (Frančula 1971). An essentially equivalent version of its formula allows weighting the areal and angular distortions (Bajeva 1987). Here, both weights are $1 / 2$ to reduce the shape distortion of continents (Kerkovits 2019):

$$
E=\sqrt{\frac{1}{S} \int_{S} \frac{\ln ^{2}(a b)+\ln ^{2}(a / b)}{2} d S}=\sqrt{\frac{1}{S} \int_{S} \ln ^{2} a+\ln ^{2} b d S}
$$

This integral was evaluated numerically using twopoint Gaussian quadrature generalized for irregular spherical polygons (Kerkovits 2017, Kerkovits, in press).

The overall distortion $E$ was minimized in the following manner: Unknown functions were approximated by fifth-degree series. Unknown coefficients of the series were determined by a modified Nelder-Mead method (Kaczmarczyk n. d.) suitable for a bigger number of parameters. Canters (2002) applied a similar method for such tasks.

In sections $4-8$, various types of polyazimuthal projections are developed. All possible projections are adapted using the methodology described previously for two study areas (cf. Figure 2):

- The Northern Atlantic Ocean and the Arctic Sea together with $\lambda_{m}=-45^{\circ}$ (Displayed in green on Figure 2)

- Southern lands: Antarctica, Australia, and New Zealand with $\lambda_{m}=135^{\circ}$ (The colatitude $\delta$ is measured from the South Pole. Violet area on Figure 2) The coordinates of the vertices defining these polygons were extracted from shapefiles found in the Natural Earth database. The reader may find the resulting low-distortion projections in section 9 .

\section{General Aphylactic Polyazimuthal Projection}

The easiest task is to develop low-distortion polyazimuthal mapping without any further expectations concerning the distortions and the properties of the graticule. $\varrho$ and $c$ are respectively odd and even functions of one variable. Any smooth function can be approximated using Maclaurin series:

$$
\begin{aligned}
& \varrho=r_{1} \delta+r_{3} \delta^{3}+r_{5} \delta^{5}+\cdots \\
& c=\varsigma_{0}+\varsigma_{2} \delta^{2}+\varsigma_{4} \delta^{4}+\cdots
\end{aligned}
$$

where $\varsigma_{0}$ results in only a translation of the whole map, it can be safely disregarded without loss of generality.

To determine $\omega$, the $\chi$ auxiliary angle is used as defined in section 2. It is a periodic odd function of $\lambda$, so a Fourier sine series can approximate it to any desired accuracy. Being a bivariate function, the Fourier coefficients of $\chi$ are not constants, rather functions of $\delta$. Maclaurin series are used for the approximation of the Fourier coefficients:

$$
\begin{aligned}
& \chi=\left(w_{01}+w_{11} \delta+w_{21} \delta^{2}+\cdots\right) \sin \lambda \\
& +\left(w_{02}+w_{12} \delta+w_{22} \delta^{2}+\cdots\right) \sin (2 \lambda) \\
& +\left(w_{03}+w_{13} \delta+w_{23} \delta^{2}+\cdots\right) \sin (3 \lambda)+\cdots
\end{aligned}
$$

To gain smooth representation at the pole, some of the $w_{i j}$ coefficients must be zero:

- The linear scale must be any times differentiable on bimeridians crossing the pole. The odd $\varrho$ and

KiG No. 32, Vol. 18, 2019, https://doi.org/10.32909/kg.18.32.2 - - 


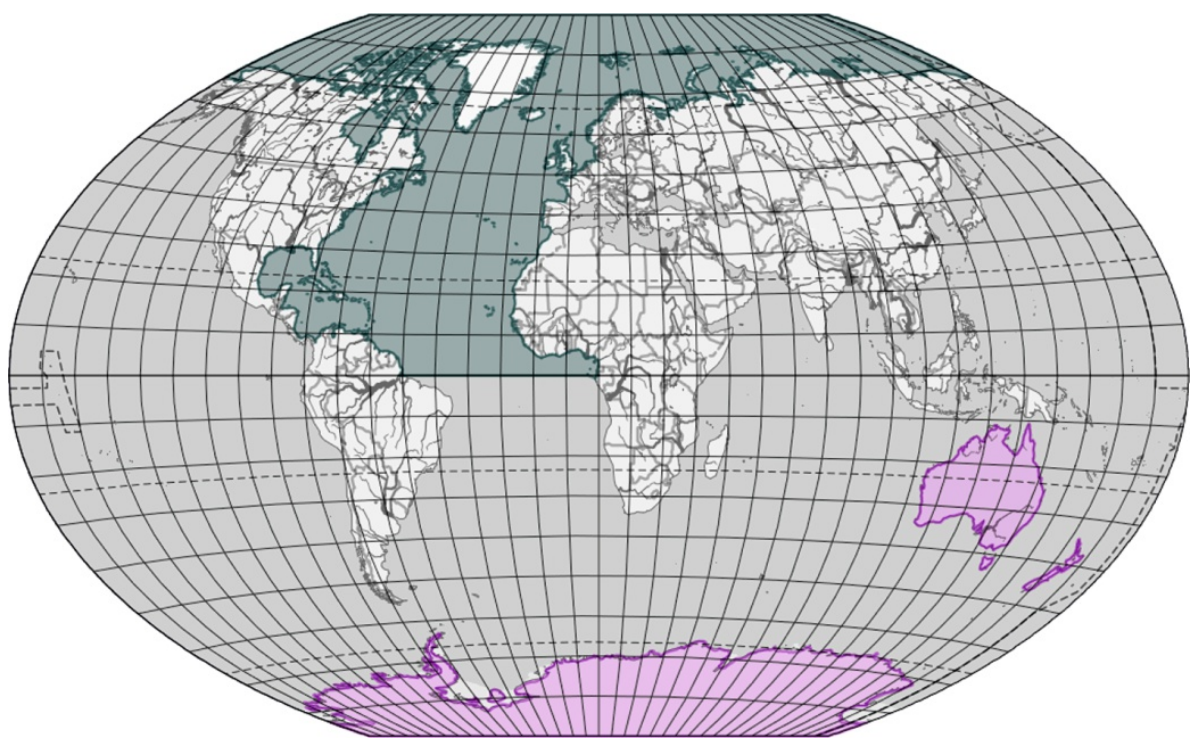

Slika 2. Područja za koja su izvedene poliazimutne projekcije.

Fig. 2 Study areas for the developed polyazimuthal map projections.

$k=\frac{\sqrt{\left(\frac{\partial x}{\partial \lambda}\right)^{2}+\left(\frac{\partial y}{\partial \lambda}\right)^{2}}}{\cos \varphi}=\frac{\varrho \frac{\partial \omega}{\partial \lambda}}{\sin \delta}$.

Konačno, poluosi $a \mathrm{i} b$ Tissotove indikatrise su:

$$
\begin{aligned}
& a=\frac{\sqrt{h^{2}+k^{2}+2 h k \sin \vartheta}+\sqrt{h^{2}+k^{2}-2 h k \sin \vartheta}}{2} \\
& b=\frac{\sqrt{h^{2}+k^{2}+2 h k \sin \vartheta}-\sqrt{h^{2}+k^{2}-2 h k \sin \vartheta}}{2} .
\end{aligned}
$$

Cjelokupna vrijednost distorzije koja sadrži učinke distorzija površina i kutova na području $S$ može se učinkovito mjeriti kriterijem Airy-Kavrajskoga (Frančula 1971). Ekvivalentna verzija te formule omogućava davanje težina površinskim i kutnim distorzijama (Bajeva 1987). U ovom su radu obje težine $1 / 2$ kako bi se smanjila distorzija oblika kontinenata (Kerkovits 2019):

$E=\sqrt{\frac{1}{S} \int_{S} \frac{\ln ^{2}(a b)+\ln ^{2}(a / b)}{2}} d S=\sqrt{\frac{1}{S} \int_{S} \ln ^{2} a+\ln ^{2} b d S}$. (10)

Taj je integral procijenjen upotrebom numeričke integracije Gaussovom metodom (2 point Gauss Quadrature), poopćene na neregularne sferne poligone (Kerkovits 2017, Kerkovits u tisku).

Cjelokupna distorzija $E$ je minimizirana na sljedeći način: nepoznate funkcije aproksimirane su redovima petog stupnja. Nepoznati koeficijenti redova određeni su modificiranom metodom Neldera i Meada (Nelder-Mead method, Kaczmarczyk, n. d.) pogodnom za veći broj parametara. Canters (2002) je za takve zadatke primijenio sličnu metodu.

U poglavljima 4-8, izvedeni su različiti tipovi poliazimutnih projekcija. Sve moguće projekcije prilagođene su upotrebom prethodno opisane metodologije za dva područja (vidi sliku 2):

- Sjeverni Atlantski ocean i Arktičko more uz $\lambda_{m}=-45^{\circ}$ (Prikazano zelenim na slici 2)

- južne zemlje: Antarktika, Australija i Novi Zeland uz $\lambda_{m}=135^{\circ}$ (Zenitna daljina $\delta$ mjerena je od Južnog pola; ljubičasto područje na slici 2).

Koordinate vrhova koji definiraju te poligone izvađene su iz datoteka (shapefiles) u bazi Natural Earth. Rezultirajuće projekcije s malim distorzijama nalaze se u poglavlju 9.

\section{Opća afilaktička poliazimutna projekcija}

Najjednostavniji je zadatak dobiti poliazimutno preslikavanje s malim distorzijama bez dodatnih očekivanja na distorzije i svojstva kartografske mreže. $\varrho$ i $c$ su neparna, odnosno parna funkcija jedne varijable. Svaka se glatka funkcija može aproksimirati upotrebom Maclaurinova reda:

$$
\begin{aligned}
& \varrho=r_{1} \delta+r_{3} \delta^{3}+r_{5} \delta^{5}+\cdots \\
& c=\varsigma_{0}+\varsigma_{2} \delta^{2}+\varsigma_{4} \delta^{4}+\cdots
\end{aligned}
$$

gdje $\varsigma_{0}$ znači translaciju cijele karte pa se može zanemariti bez gubitka općenitosti. 
even $c$ already ensure this; there is no additional requirement on $\omega$.

- The curvature of bimeridians must be any times differentiable. This may be fulfilled by setting $w_{i j}=0$ if $i+j$ is odd.

- The high-order mixed partial derivatives of the projection formulae must be independent of $\lambda$ at the pole. Thus, it is necessary to set $w_{i j}=0$ if $i<j$. This also enforces the conformity at the pole mentioned in section 2, because $w_{0 j}$ is now zero for all $j$. Therefore, $\chi=0$ at the pole.

These additional requirements generate the following function for omega:

$$
\begin{aligned}
& \omega=\lambda+\chi=\lambda+\left(w_{11} \delta+w_{31} \delta^{3}+\cdots\right) \sin \lambda \\
& +\left(w_{22} \delta^{2}+w_{42} \delta^{4}+\cdots\right) \sin (2 \lambda) \\
& +\left(w_{33} \delta^{3}+\cdots\right) \sin (3 \lambda)+\left(w_{44} \delta^{4}+\cdots\right) \sin (4 \lambda)+\cdots
\end{aligned}
$$

This series is now truncated for five-degree terms (if $\sin (n \lambda)$ is considered as a linear factor), consistently with the other two functions. The inclusion of the fifth power in $\delta$ would not influence the fifth-order directional derivatives at the pole. Eleven coefficients remain undetermined for a fifth-degree approximation of the optimal map projection. See the results in section 9 .

\section{Equal-area Polyazimuthal Projection}

To develop an equal-area or equivalent projection, the equation $a b=h k \sin \vartheta=1$ must be solved. After substituting formulae (5) - (7) and simplifying the result one may yield

$$
\frac{\varrho \frac{\partial \omega}{\partial \lambda}}{\sin \delta}\left(\frac{d \varrho}{d \delta}-\cos \omega \frac{d c}{d \delta}\right)=1 .
$$

Knowing that the area of a spherical cap must be equal to the area of the corresponding circle on the map:

$$
2 \pi(1-\cos \delta)=\varrho^{2} \pi
$$

Rearranged using half-angle formulae:

$$
\varrho=\sqrt{2\left(1-\cos ^{2} \frac{\delta}{2}+\sin ^{2} \frac{\delta}{2}\right)}=2 \sin \frac{\delta}{2} .
$$

This is substituted into formula (15):

$$
\begin{aligned}
& \frac{2 \sin \frac{\delta}{2} \frac{\partial \omega}{\partial \lambda}}{\sin \delta}\left(\cos \frac{\delta}{2}-\cos \omega \frac{d c}{d \delta}\right)= \\
& \frac{\partial \omega}{\partial \lambda}\left(1-\frac{2 \sin \frac{\delta}{2} \cos \omega}{\sin \delta} \frac{d c}{d \delta}\right)=1
\end{aligned}
$$

Until this point, the derivation of the equivalent polyazimuthal projection can also be found in Tolstova (1981). Solving the separable partial differential equation for $\lambda$ :

$$
\lambda=\omega-\frac{d c}{d \delta} \frac{\varrho \sin \omega}{\sin \delta}+f(\delta)
$$

where the integration constant $f(\delta)$ must be zero to get an odd function for $\omega$.

If the function $c(\delta)$ is known, formula (19) uniquely defines the function $\omega(\delta, \lambda)$. Let us approximate the unknown $c(\delta)$ again by the following even polynomial:

$$
c=\varsigma_{2} \delta^{2}+\varsigma_{4} \delta^{4}+\cdots
$$

Substitution back into formula (19) yields:

$$
\lambda=\omega-\frac{\varrho\left(2 \varsigma_{2} \delta+4 \varsigma_{4} \delta^{3}+\cdots\right)}{\sin \delta} \sin \omega .
$$

The angle $\omega$ cannot be expressed in a closed form, only as an implicit function. Numerical methods must be used to obtain approximate values. Two coefficients remained undetermined for the fifth-degree optimization.

\section{Orthogonal Polyazimuthal Projection}

A map projection is orthogonal if all meridians and parallels cross at right angles, i. e. $\cot \vartheta=0$ (Györffy, 2002):

$$
\frac{d c}{d \delta} \sin \omega+\varrho \frac{d \omega}{d \delta}=0 .
$$

Solving the separable partial differential equation for $\omega$ :

$$
\ln \tan \frac{\omega}{2}=-\int \frac{d c}{\varrho} d \delta+f(\lambda) .
$$

The integration constant $f(\lambda)$ can be determined from the fact that the projection must be conformal

KiG No. 32, Vol. 18, 2019, https://doi.org/10.32909/kg.18.32.2 - - 
Da bismo odredili $\omega$, upotrijebit ćemo pomoćni kut $\chi$ definiran u poglavlju 2. To je periodična neparna funkcija od $\lambda$ pa se može aproksimirati Fourierovim redom sinusa do bilo koje željene točnosti. Zbog funkcije dviju varijabli, Fourierovi koeficijenti $\chi$ nisu konstante, već funkcije od $\delta$. Maclaurinovi redovi služe za aproksimaciju Fourierovih koeficijenata:

$$
\begin{aligned}
& \chi=\left(w_{01}+w_{11} \delta+w_{21} \delta^{2}+\cdots\right) \sin \lambda \\
& +\left(w_{02}+w_{12} \delta+w_{22} \delta^{2}+\cdots\right) \sin (2 \lambda) \\
& +\left(w_{03}+w_{13} \delta+w_{23} \delta^{2}+\cdots\right) \sin (3 \lambda)+\cdots
\end{aligned}
$$

Da bi se dobio glatki prikaz u polu, neki od koeficijenata $w_{i j}$ moraju biti jednaki nuli:

- Linearno mjerilo mora biti višestruko diferencijabilno na bimeridijanima koji prolaze polom. Neparna funkcija $\varrho$ i parna $c$ to već osiguravaju. Nema dodatnih zahtjeva na $\omega$.

- Zakrivljenost bimeridijana mora biti višestruko diferencijabilna. To se može postići stavljanjem $w_{i j}=0$ ako je $i+j$ neparno.

- Miješane parcijalne derivacije višeg reda u formulama projekcije moraju biti neovisne u polu o $\lambda$. Dakle, nužno je staviti $w_{i j}=0$ ako je $i<j$. To ima za posljedicu konformnost u polu, spomenutu u poglavlju 2, jer je sada $w_{0 j}$ jednako nuli za sve $j$. Dakle, $\chi=0$ u polu.

Navedni dodatni zahtjevi generiraju ovakav oblik funkcije za omega:

$$
\begin{aligned}
& \omega=\lambda+\chi=\lambda+\left(w_{11} \delta+w_{31} \delta^{3}+\cdots\right) \sin \lambda \\
& +\left(w_{22} \delta^{2}+w_{42} \delta^{4}+\cdots\right) \sin (2 \lambda) \\
& +\left(w_{33} \delta^{3}+\cdots\right) \sin (3 \lambda)+\left(w_{44} \delta^{4}+\cdots\right) \sin (4 \lambda)+\cdots
\end{aligned}
$$

Ti su redovi sada skraćeni do članova 5. reda (ako se $\sin (n \lambda)$ smatra linearnim faktorom), konzistentno s dvjema drugim funkcijama. Uključivanje članova s petom potencijom od $\delta$ ne bi utjecalo na usmjerenu derivaciju 5. reda u polu. Jedanaest koeficijenata ostaje neodređeno za aproksimaciju 5. stupnja optimalne projekcije. Rezultati se mogu vidjeti u poglavlju 9.

\section{Ekvivalentna poliazimutna projekcija}

Da bismo izveli ekvivalentnu projekciju, potrebno je riješiti jednadžbu $a b=h k \sin \vartheta=1$. Uvrštavanjem formula (5) - (7) i pojednostavljivanjem rezultata dobije se

$$
\frac{\varrho \frac{\partial \omega}{\partial \lambda}}{\sin \delta}\left(\frac{d \varrho}{d \delta}-\cos \omega \frac{d c}{d \delta}\right)=1 .
$$

Znajući da površina sferne kapice treba biti jednaka površini odgovarajućeg kruga na karti, mora biti:

$$
2 \pi(1-\cos \delta)=\varrho^{2} \pi .
$$

Preuredivši upotrebom formula polovičnog kuta, dobije se:

$$
\varrho=\sqrt{2\left(1-\cos ^{2} \frac{\delta}{2}+\sin ^{2} \frac{\delta}{2}\right)}=2 \sin \frac{\delta}{2} .
$$

Uvrstimo li to u (15), dobijemo:

$$
\begin{aligned}
& \frac{2 \sin \frac{\delta}{2} \frac{\partial \omega}{\partial \lambda}}{\sin \delta}\left(\cos \frac{\delta}{2}-\cos \omega \frac{d c}{d \delta}\right)= \\
& \frac{\partial \omega}{\partial \lambda}\left(1-\frac{2 \sin \frac{\delta}{2} \cos \omega}{\sin \delta} \frac{d c}{d \delta}\right)=1 .
\end{aligned}
$$

Do ovoga se mjesta izvođenja ekvivalentne poliazimutne projekcije može također doći kod Tolstove (1981). Rješavajući metodom separacije parcijalnu diferencijalnu jednadžbu po $\lambda$ :

$$
\lambda=\omega-\frac{d c}{d \delta} \frac{\varrho \sin \omega}{\sin \delta}+f(\delta)
$$

gdje integracijska konstanta $f(\delta)$ mora biti jednaka nuli da bi se dobila neparna funkcija $\omega$.

Ako je funkcija $c(\delta)$ poznata, formula (19) na jedinstveni način definira funkciju $\omega(\delta, \lambda)$. Aproksimirajmo nepoznatu funkciju $c(\delta)$ opet s pomoću ovog parnog polinoma:

$$
c=\varsigma_{2} \delta^{2}+\varsigma_{4} \delta^{4}+\cdots
$$

Supstituirajući natrag u formulu (19), dobijemo:

$$
\lambda=\omega-\frac{\varrho\left(2 \varsigma_{2} \delta+4 \varsigma_{4} \delta^{3}+\cdots\right)}{\sin \delta} \sin \omega .
$$

Kut $\omega$ se ne može izraziti u zatvorenom obliku, već samo kao implicitna funkcija. Da bi se dobile približne vrijednosti, potrebno je upotrijebiti numeričke metode. Uz optimizaciju 5. stupnja ostaju neodređena dva koeficijenta.

\section{Ortogonalna poliazimutna projekcija}

Kartografska projekcija je ortogonalna ako se slike svih meridijana i paralela sijeku pod pravim kutom, tj. ako je $\cot \vartheta=0$ (Györffy 2002):

$$
\frac{d c}{d \delta} \sin \omega+\varrho \frac{d \omega}{d \delta}=0 .
$$


at the pole. That is if $\delta=0$ then $\omega=\lambda$. The only possible function satisfying it is

$$
f=\ln \tan \frac{\lambda}{2} .
$$

Two functions, $\varrho$ and $c$ are undetermined. The familiar approximation is used:

$$
\begin{aligned}
& \varrho=r_{1} \delta+r_{3} \delta^{3}+r_{5} \delta^{5}+\cdots \\
& c=\varsigma_{2} \delta^{2}+\varsigma_{4} \delta^{4}+\cdots
\end{aligned}
$$

Substituting into formula (23) and cancelling $\delta$ in the integrand:

$$
\ln \tan \frac{\omega}{2}=-\int \frac{2 \varsigma_{2}+4 \varsigma_{4} \delta^{2}+\cdots}{r_{1}+r_{3} \delta^{2}+r_{5} \delta^{4}+\cdots} d \delta+\ln \tan \frac{\lambda}{2} .
$$

The antiderivative of a rational function is always expressible in terms of standard mathematical functions. $r_{1}$ is positive, otherwise, the function $\varrho$ would not be strictly increasing near the pole, which would result in an unacceptable map. It is reasonable to assume, that the only real root of $\varrho$ is at $\delta=0$, otherwise a full parallel would collapse into a single point on the map. Thus, the discriminant $r_{3}^{2}-4 r_{1} r_{5}$ is negative. Therefore, $r_{5}$ must be positive. These assumptions make it possible to decompose the integrand into the following partial fractions:

$$
\frac{2 \varsigma_{2}+4 \varsigma_{4} \delta^{2}}{r_{1}+r_{3} \delta^{2}+r_{5} \delta^{4}}=\frac{p_{1} \delta+p_{2}}{\delta^{2}+p_{3} \delta+p_{4}}+\frac{p_{5} \delta+p_{6}}{\delta^{2}+p_{7} \delta+p_{8}}
$$

where the coefficients $p_{1}$ to $p_{8}$ can be expressed by solving a quartic equation. The long derivation is omitted due to lack of space. The correct root is chosen from the four solutions so that no negative numbers appear under square roots:

$$
\begin{aligned}
& p_{1}=-p_{5}=\frac{\sqrt{r_{5}} \varsigma_{2}-2 \sqrt{r_{1}} \varsigma_{4}}{\sqrt{r_{1} r_{5}\left(2 \sqrt{r_{1} r_{5}}-r_{3}\right)}} \\
& p_{2}=p_{6}=\frac{\varsigma_{2}}{\sqrt{r_{1} r_{5}}} \\
& p_{3}=-p_{7}=\sqrt{\frac{2 \sqrt{r_{1} r_{5}}-r_{3}}{r_{5}}} \\
& p_{4}=p_{8}=\sqrt{\frac{r_{1}}{r_{5}}} .
\end{aligned}
$$

The right side of the equation (28) is easy to integrate. Lets substitute its antiderivative into formula (27) to gain the final solution for $\omega$ :

$$
\begin{aligned}
& \ln \tan \frac{\omega}{2}=-\frac{p_{1} p_{3}-2 p_{2}}{\sqrt{4 p_{4}-p_{3}^{2}}}\left(\arctan \frac{p_{3}-2 \delta}{\sqrt{4 p_{4}-p_{3}^{2}}}-\right. \\
& \left.\arctan \frac{p_{3}+2 \delta}{\sqrt{4 p_{4}-p_{3}^{2}}}\right)-\frac{p_{1}}{2} \ln \frac{\delta^{2}+p_{3} \delta+p_{4}}{\delta^{2}-p_{3} \delta+p_{4}}+\ln \tan \frac{\lambda}{2} .
\end{aligned}
$$

Five coefficients $\left(r_{1}, r_{3}, r_{5}, \varsigma_{2}, \varsigma_{4}\right)$ remained undetermined for further optimization.

\section{Conformal Polyazimuthal Projection}

Tolstova (1981) stated that a necessary and sufficient condition for a polyazimuthal map projection to be conformal is to fulfil both formula (22) for orthogonality and $h=k$ :

$$
\frac{d \varrho}{d \delta}-\cos \omega \frac{d c}{d \delta}=\frac{\varrho}{\sin \delta} \frac{\partial \omega}{\partial \lambda} .
$$

The first-order partial differential equation (34) is hard to solve because it is not separable. Furthermore, both sides depend on $\lambda$ through $\omega$, while the unknown functions $c$ and $\varrho$ may only depend on $\delta$. Tolstova (1981) listed a particular solution to this equation in her article. Careful investigation of her formulae shows, however, that she only found a complicated way to derive a transverse stereographic projection with the bimeridian of $\pm 90^{\circ}$ longitude as its standard line.

Examining polyconic projections, Adams (1934) could solve the same set of differential equations halfcentury earlier. After long pages of derivation, he proved that conformal projections with circular parallels must also have circular meridians. In other words, the general solution to this set of differential equations is the family of the Lagrange map projection.

Conformal polyazimuthal projections must be holomorphic (i. e. conformal) at the pole (cf. chapter 2). On the other hand, the Lagrange map projection family is generally singular (not conformal) at the poles. Therefore, only a subset of the projection family is appropriate, in that meridians reach the pole with undistorted angles. Consequently, the only possible conformal polyazimuthal projection is the oblique stereographic one.

The oblique stereographic projection maps all spherical circles to circles (Adams 1934); it satisfies the

KiG No. 32, Vol. 18, 2019, https://doi.org/10.32909/kg.18.32.2 - - 
Rješenje separabilne parcijalne diferencijalne jednadžbe po $\omega$ je:

$$
\ln \tan \frac{\omega}{2}=-\int \frac{\frac{d c}{d \delta}}{\varrho} d \delta+f(\lambda) .
$$

Integracijska konstanta $f(\lambda)$ može se odrediti iz činjenice da projekcija mora biti konformna u polu. To jest, ako je $\delta=0$, onda je $\omega=\lambda$. Jedina funkcija koja to zadovoljava je

$$
f=\ln \tan \frac{\lambda}{2} .
$$

Dvije funkcije $\varrho$ i c su neodređene. Upotrijebit ćemo poznatu aproksimaciju:

$$
\begin{aligned}
& \varrho=r_{1} \delta+r_{3} \delta^{3}+r_{5} \delta^{5}+\cdots \\
& c=\varsigma_{2} \delta^{2}+\varsigma_{4} \delta^{4}+\cdots .
\end{aligned}
$$

Uvrštavanjem u formulu (23), dobit ćemo:

$$
\ln \tan \frac{\omega}{2}=-\int \frac{2 \varsigma_{2}+4 \varsigma_{4} \delta^{2}+\cdots}{r_{1}+r_{3} \delta^{2}+r_{5} \delta^{4}+\cdots} d \delta+\ln \tan \frac{\lambda}{2} .
$$

Integral racionalne funkcije uvijek se može izraziti s pomoću standardnih matematičkih funkcija. $r_{1}$ je pozitivan jer u protivnom funkcija $\varrho$ ne bi bila striktno rastuća u blizini pola, što bi rezultiralo neprihvatljivom kartom. Razumno je pretpostaviti da postoji samo jedan korijen od $\varrho$ za $\delta=0$, jer bi se u protivnom cijela paralela stisnula u točku. Dakle, diskriminanta $r_{3}^{2}-4 r_{1} r_{5}$ je negativna. Prema tome, $r_{5}$ mora biti pozitivan. Te pretpostavke omogućuju rastavljanje integrala u parcijalne razlomke:

$$
\frac{2 \varsigma_{2}+4 \varsigma_{4} \delta^{2}}{r_{1}+r_{3} \delta^{2}+r_{5} \delta^{4}}=\frac{p_{1} \delta+p_{2}}{\delta^{2}+p_{3} \delta+p_{4}}+\frac{p_{5} \delta+p_{6}}{\delta^{2}+p_{7} \delta+p_{8}}
$$

gdje se koeficijenti $p_{1}$ do $p_{8}$ mogu izraziti rješavanjem jednadžbe četvrtog stupnja. Dugački izvod izostavljamo. Ispravan je korijen odabran između četiriju rješenja tako da se ispod kvadratnih korijena ne pojavljuju negativni brojevi:

$$
\begin{aligned}
& p_{1}=-p_{5}=\frac{\sqrt{r_{5}} \varsigma_{2}-2 \sqrt{r_{1}} \varsigma_{4}}{\sqrt{r_{1} r_{5}\left(2 \sqrt{r_{1} r_{5}}-r_{3}\right)}} \\
& p_{2}=p_{6}=\frac{\varsigma_{2}}{\sqrt{r_{1} r_{5}}} \\
& p_{3}=-p_{7}=\sqrt{\frac{2 \sqrt{r_{1} r_{5}}-r_{3}}{r_{5}}} .
\end{aligned}
$$

$$
p_{4}=p_{8}=\sqrt{\frac{r_{1}}{r_{5}}}
$$

Desnu je stranu jednadžbe (28) lako integrirati. Uvrstimo njezin integral $u$ formulu (27) da bismo dobili konačno rješenje za $\omega$ :

$$
\begin{aligned}
& \ln \tan \frac{\omega}{2}=-\frac{p_{1} p_{3}-2 p_{2}}{\sqrt{4 p_{4}-p_{3}^{2}}}\left(\arctan \frac{p_{3}-2 \delta}{\sqrt{4 p_{4}-p_{3}^{2}}}-\right. \\
& \left.\arctan \frac{p_{3}+2 \delta}{\sqrt{4 p_{4}-p_{3}^{2}}}\right)-\frac{p_{1}}{2} \ln \frac{\delta^{2}+p_{3} \delta+p_{4}}{\delta^{2}-p_{3} \delta+p_{4}}+\ln \tan \frac{\lambda}{2} .
\end{aligned}
$$

Pet koeficijenata $\left(r_{1}, r_{3}, r_{5}, \varsigma_{2}, \varsigma_{4}\right)$ ostaje neodređeno za daljnje optimiranje.

\section{Konformna poliazimutna projekcija}

Prema Tolstovoj (1981) nužan i dovoljan uvjet da bi poliazimutna projekcija bila konformna je da su ispunjene formule (22) za ortogonalnost i $h=k$ :

$$
\frac{d \varrho}{d \delta}-\cos \omega \frac{d c}{d \delta}=\frac{\varrho}{\sin \delta} \frac{\partial \omega}{\partial \lambda} .
$$

Parcijalnu diferencijalnu jednadžbu prvog reda (34) nije lako riješiti jer nije separabilna. Nadalje, obje strane ovise o $\lambda$ preko $\omega$, dok nepoznate funkcije $c$ i $\varrho$ mogu ovisiti samo o $\delta$. Tolstova (1981) je u svojem članku dala partikularno rješenje te jednadžbe. Međutim, pažljivo ispitivanje njezinih formula pokazuje da je ona našla samo kompliciran način izvoda za poprečnu stereografsku projekciju s bimeridijanom $\pm 90^{\circ}$ geografske dužine kao standardnom linijom. Istražujući polikonusne projekcije, Adams (1934) je pola stoljeća ranije riješio isti skup diferencijalnih jednadžbi. Nakon mnogo stranica izvoda on je dokazao da konformne projekcije s kružnim paralelama moraju također imati kružne meridijane. Drugim riječima, opće je rješenje toga skupa diferencijalnih jednadžbi familija Lagrangeovih projekcija.

Konformne poliazimutne projekcije moraju u polu biti konformne (vidi poglavlje 2). S druge strane, Lagrangeove projekcije su u polovima općenito singularne (nekonformne). Dakle, jedini podskup te familije koji odgovara je onaj kod kojega se meridijani u polu sijeku pod kutom bez distorzije, a jedina moguća konformna poliazimutna projekcija je kosa stereografska.

Kosa stereografska projekcija preslikava sve sferne kružnice u kružnice (Adams 1934) - ona zadovoljava zahtjeve postavljene u definiciji poliazimutnih projekcija. Međutim, nju ne bi trebalo smatrati 
Table 1 Optimal coefficients for the Northern Atlantic Ocean.

Tablica 1. Optimalni koeficijenti za Sjeverni Atlantski ocean.

\begin{tabular}{|c|c|c|c|c|c|c|c|c|c|c|c|c|}
\hline $\begin{array}{l}\text { Projection } \\
\text { Projekcija }\end{array}$ & $r_{1}$ & $r_{3}$ & $r_{5}$ & $\varsigma_{2}$ & $\varsigma_{4}$ & $w_{11}$ & $w_{22}$ & $w_{31}$ & $w_{33}$ & $w_{42}$ & $w_{44}$ & E \\
\hline Aphylactic & 0.995403 & 0.091755 & 0.016412 & 0.049418 & 0.067848 & -0.122384 & -0.100047 & -0.091506 & 0.046000 & 0.022864 & -0.009933 & 0.053943 \\
\hline Afilaktička & 0,995403 & 0,091755 & 0,016412 & 0,049418 & 0,067848 & $-0,122384$ & $-0,100047$ & $-0,091506$ & 0,046000 & 0,022864 & $-0,009933$ & 0,053943 \\
\hline Equal-area & - & - & - & -0.079795 & 0.003259 & - & - & - & - & - & - & 0.089981 \\
\hline Ekvivalentna & - & - & - & $-0,079795$ & 0,003259 & - & - & - & - & - & - & 0,089981 \\
\hline Orthogonal & 0.965002 & 0.331444 & 0.085915 & 0.106875 & 0.302636 & - & - & - & - & - & - & 0.066337 \\
\hline Ortogonalna & 0,965002 & 0,331444 & 0,085915 & 0,106875 & 0,302636 & - & - & - & - & - & - & 0,066337 \\
\hline Equidistant & - & - & - & $-0.130302-$ & -0.045537 & - & - & - & - & - & - & 0.170722 \\
\hline Ekvidistantna & - & - & - & $-0,130302-$ & $-0,045537$ & - & - & - & - & - & - & 0,170722 \\
\hline
\end{tabular}

requirements imposed by the definition of polyazimuthal projections. However, it should not be considered as a separate polyazimuthal projection, rather the properties of the simplest aspect should be examined (Wray 1974, Lapaine \& Frančula 2016). Thus, it is rather an azimuthal projection than a polyazimuthal one. There is no conformal, strictly polyazimuthal projection.

\section{Polyazimuthal Projection with Equidistant Parallels}

Tolstova (1981) indicated that it is possible to develop a polyazimuthal mapping with true-scale parallels. The linear scale along parallels must be unit $(k=1)$ :

$$
\frac{\varrho \frac{\partial \omega}{\partial \lambda}}{\sin \delta}=1 .
$$

Furthermore, the perimeter of the mapped parallels must be equal to their spherical length:

$2 \varrho \pi=2 \pi \sin \delta$,

That is

$\varrho=\sin \delta$.

Substitute (37) into (35):

$\frac{\partial \omega}{\partial \lambda}=1$.

Integrate with respect to $\lambda$ :

$\omega=\lambda+f(\delta)$,

where the integration constant $f(\delta)$ is zero to obtain an odd function for $\omega$.

The function $c$ is yet undetermined, the usual approximation is used:

$$
c=\varsigma_{2} \delta^{2}+\varsigma_{4} \delta^{4}+\cdots .
$$

Two coefficients can be used for further optimization.

\section{Practical Application of Polyazimuthal Mappings}

The four map projections (i. e. the aphylactic, equivalent, orthogonal, and equidistant ones) were optimized using the methodology described in section 3. Results of the optimization may be found in Table 1 and on Figure 3 for the Northern Atlantic Ocean. Table 2 and Figure 4 report the results for the southern continents. Distortion isolines are drawn onto the left and right halves of the map separately to enhance readability; these lines are symmetrical to the vertical axis. Green lines demonstrate the maximum angular deviation $2 \arcsin [(a-b) /(a+b)]$ and violet lines display the areal scale $a b$.

The figures clearly show that polyazimuthal mappings are appropriate for traditional cartography. Circular parallels result in high aesthetic value. This advantage is only present in normal aspect. Thus, polyazimuthal mappings are rather recommended without graticule transformation. Furthermore, these projections can depict the anti-meridian uninterrupted.

As it is expected, the distortion values $(E)$ show that the general aphylactic projection is the least distorted. The orthogonal variant is a bit worse but the aesthetical value of the orthogonal graticule compensates for this effect. Should the map thematic require equivalency, one may use the equal-area variant with its still favourable distortions, comparable to the orthogonal one. The equal-area mapping is suggested because its distortions are only visible in areas far from the mid-meridian.

The equidistant projection exhibits the worst distortion characteristics. This variant cannot depict large areas (c.f. the gaps in the figure), its distortion value is high. The usage of this variant is discouraged. This projection reduces areas considerably almost everywhere on the map.

These map projections (with the exception of the orthogonal one) accumulated most of the distortions at lower latitudes, far from the mid-meridian. One may need to exclude these parts from the map sheet when drawing maps in these projections. This may also be the reason

KiG No. 32, Vol. 18, 2019, https://doi.org/10.32909/kg.18.32.2 - - 


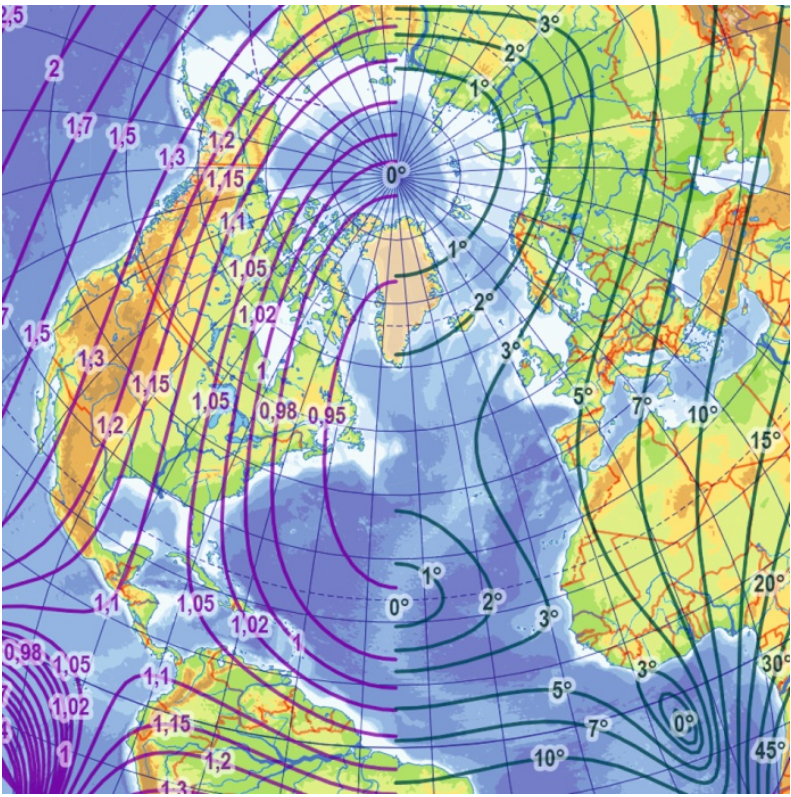

Afilaktička/ Aphylactic

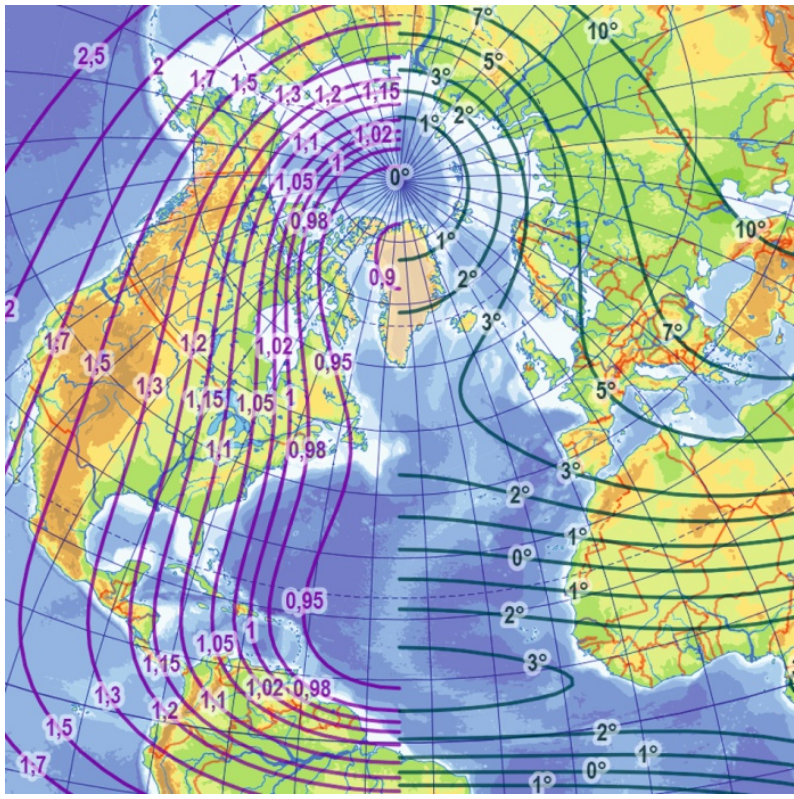

Ortogonalna/ Orthogonal

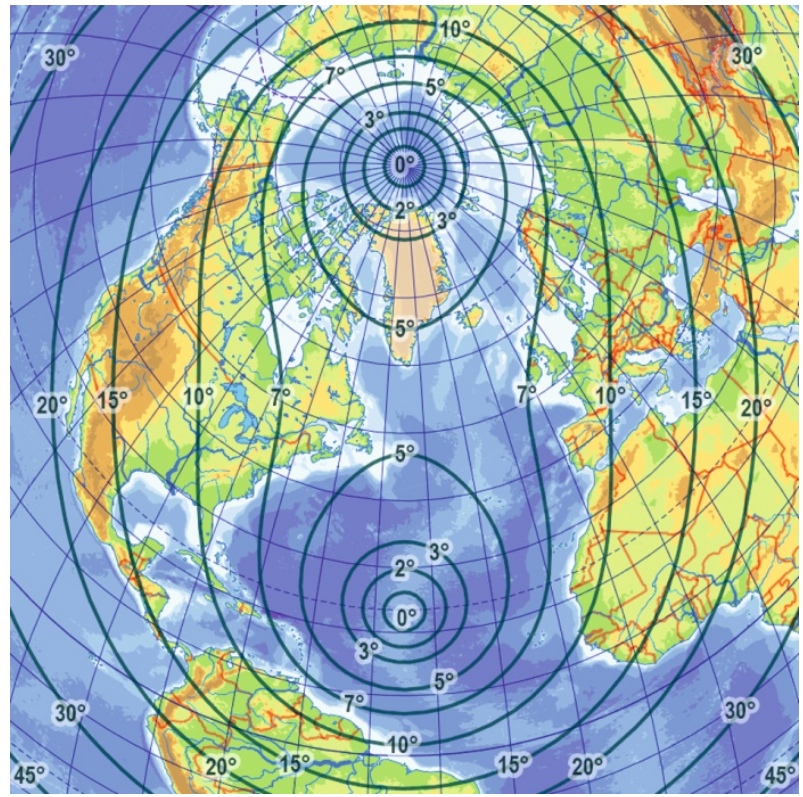

Ekvivalentna/ Equal-area

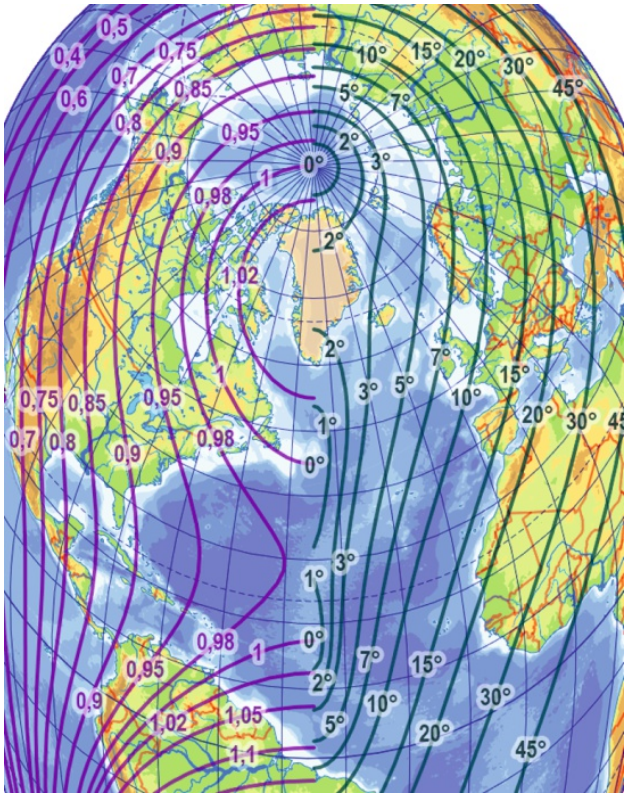

Ekvidistantna/ Equidistant

Slika 3. Optimalne poliazimutne projekcije za Sjeverni Atlantski ocean i Arktičko more.

Fig. 3 Optimal polyazimuthal projections of the Northern Atlantic Ocean and the Arctic Sea.

poliazimutnom projekcijom, bolje je istražiti svojstva najjednostavnijeg aspekta projekcije (Wray 1974, Lapaine i Frančula 2016). Dakle, to je prije azimutna, nego poliazimutna projekcija. Ne postoji konformna, strogo poliazimutna projekcija.

\section{Poliazimutna projekcija s ekvidistantnim paralelama}

Tolstova (1981) je ukazala na mogućnost izvoda poliazimutnog preslikavanja s ekvidistantnim paralelama.
Linearno mjerilo uzduž paralela mora biti jednako jedan $(k=1)$ :

$$
\frac{\varrho \frac{\partial \omega}{\partial \lambda}}{\sin \delta}=1 .
$$

Nadalje, opseg preslikanih paralela mora biti jednak njihovoj duljini na sferi:

$$
2 \varrho \pi=2 \pi \sin \delta
$$


Table 2 Optimal coefficients for the southern continents.

Tablica 2. Optimalni koeficijenti za južne kontinente.

\begin{tabular}{|c|c|c|c|c|c|c|c|c|c|c|c|c|}
\hline $\begin{array}{l}\text { Projection } \\
\text { Projekcija }\end{array}$ & $r_{1}$ & $r_{3}$ & $r_{5}$ & $\varsigma_{2}$ & $\varsigma_{4}$ & $w_{11}$ & $w_{22}$ & $w_{31}$ & $w_{33}$ & $w_{42}$ & $w_{44}$ & E \\
\hline Aphylactic & 0.986965 & 0.066843 & 0.035334 & 0.000726 & -0.094266 & 0.005879 & -0.067769 & 0.270597 & -0.012451 & 0.083305 & -0.008414 & 0.023914 \\
\hline Afilaktička & 0,986965 & 0,066843 & 0,035334 & 0,000726 & $-0,094266$ & 0,005879 & $-0,067769$ & 0,270597 & $-0,012451$ & 0,083305 & $-0,008414$ & 0,023914 \\
\hline Equal-area & - & - & - & 0.032125 & 0.012790 & - & - & - & - & - & - & 0.039807 \\
\hline Ekvivalentna & - & - & - & 0,032125 & 0,012790 & - & - & - & - & - & - & 0,039807 \\
\hline Orthogonal & 0.971476 & 0.221766 & 0.093476 & -0.066344 & -0.254108 & - & - & - & - & - & - & 0.047721 \\
\hline Ortogonalna & 0,971476 & 0,221766 & 0,093476 & $-0,066344$ & $-0,254108$ & - & - & - & - & - & - & 0,047721 \\
\hline Equidistant & - & - & - & 0.067020 & 0.070053 & - & - & - & - & - & - & 0.064120 \\
\hline Ekvidistantna & - & - & - & 0,067020 & 0,070053 & - & - & - & - & - & - & 0,064120 \\
\hline
\end{tabular}

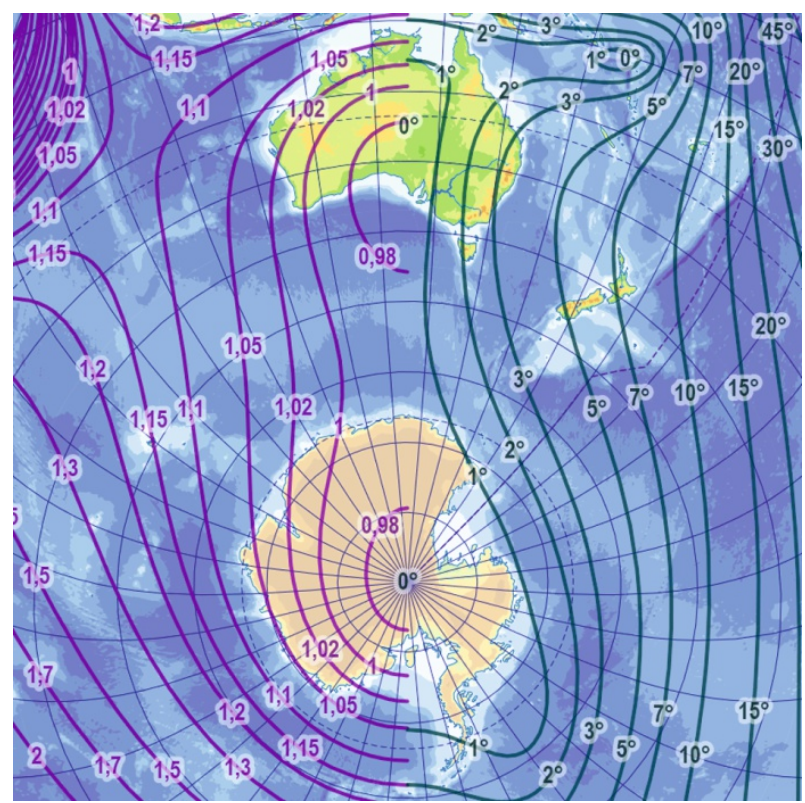

Aphylactic/ Afilaktička

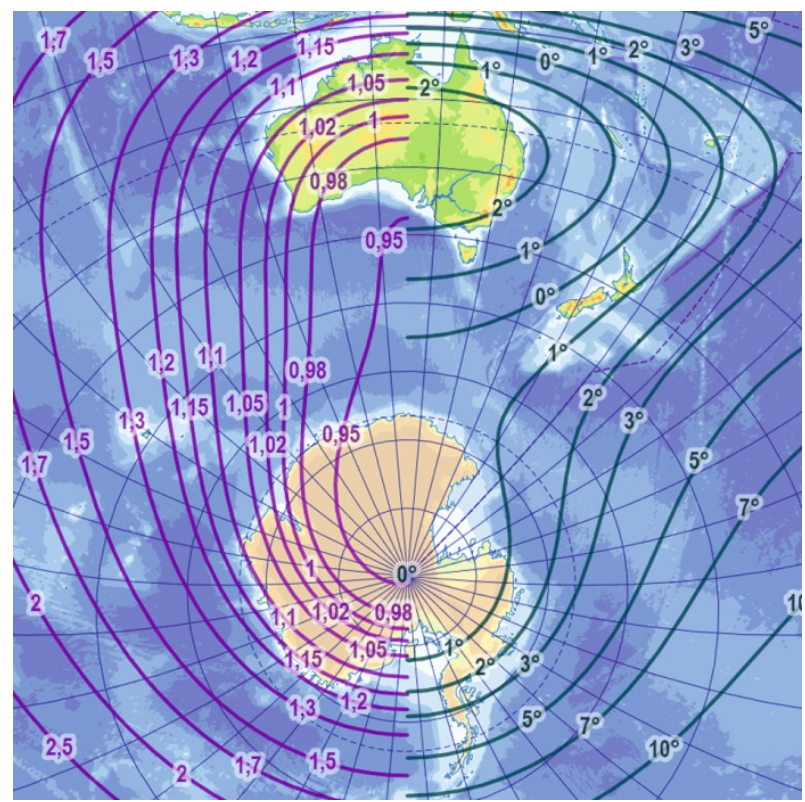

Orthogonal/ Ortogonalna

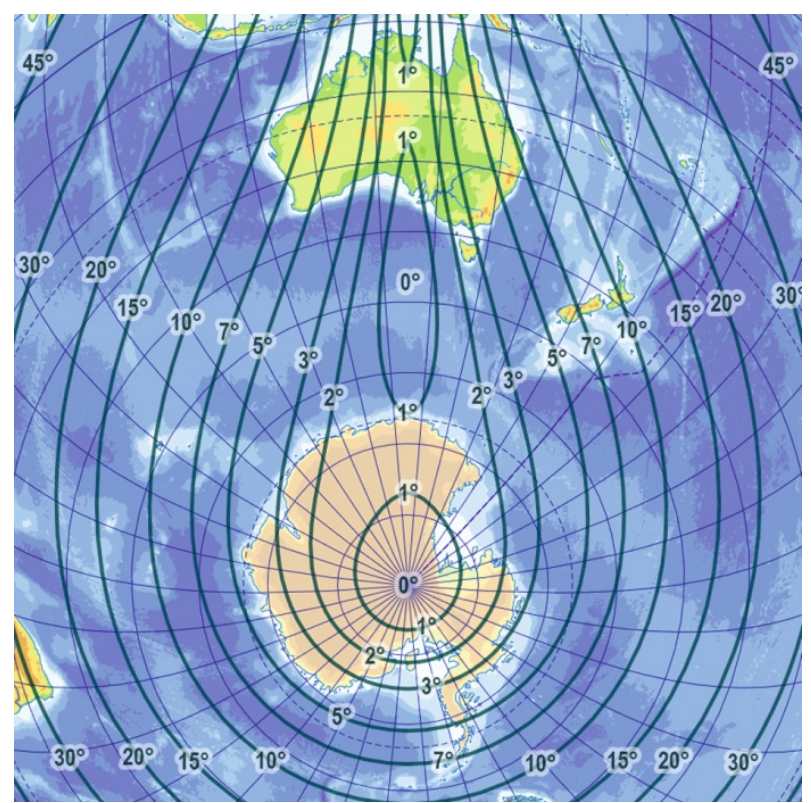

Equal-area/ Ekvivalentna

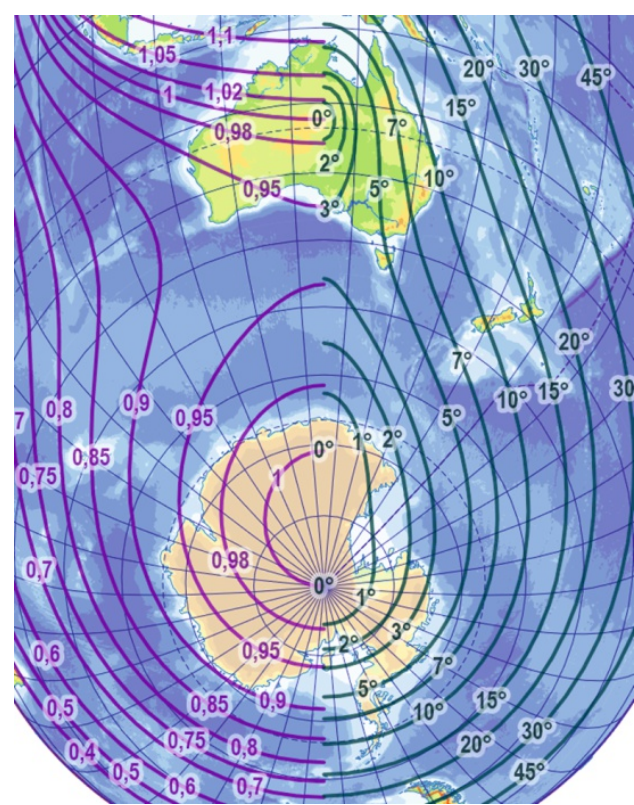

Equidistant/ Ekvidistantna

Fig 4 Optimal polyazimuthal projections of Australia, New Zealand, and Antarctica.

Slika 4. Optimalne poliazimutne projekcije Australije, Novog Zelanda i Antarktika. 
Tj.

$\varrho=\sin \delta$.

Uvrstimo li (37) u (35):

$\frac{\partial \omega}{\partial \lambda}=1$.

Integriranje po $\lambda$ daje:

$\omega=\lambda+f(\delta)$,

gdje integracijska konstanta $f(\delta)$ mora biti jednaka nuli da bi $\omega$ bila neparna funkcija. Funkcija $c$ je još neodređena pa se može upotrijebiti uobičajena aproksimacija:

$$
c=\varsigma_{2} \delta^{2}+\varsigma_{4} \delta^{4}+\cdots
$$

Dva se koeficijenta mogu upotrijebiti za daljnje optimiranje.

\section{Praktična primjena poliazimutnih preslikavanja}

Četiri projekcije, tj. afilaktička, ekvivalentna, ortogonalna i ekvidistantna su optimirane upotrebom metodologije opisane u poglavlju 3. Rezultati optimiranja nalaze se u tablici 1 i na slici 3 za Sjeverni Atlantski ocean. Tablica 2 i slika 4 prikazuju rezultate za južne kontinente. Izolinije distorzija iscrtane su na lijevoj i desnoj polovici karte kako bi se povećala čitljivost; te su linije simetrične u odnosu na vertikalnu os. Zelene linije prikazuju maksimalne distorzije kutova 2 arc$\sin [(a-b) /(a+b)]$, a ljubičaste površinsko mjerilo $a b$.

slike jasno pokazuju da su poliazimutna preslikavanja pogodna za tradicionalnu kartografiju. Kružne paralele daju veliku estetsku vrijednost. Ta je prednost prisutna samo u uspravnom aspektu projekcije. Dakle, poliazimutna se preslikavanja preporučuju bez transformacije kartografske mreže. Nadalje, te projekcije mogu prikazati antimeridijan bez prekida.

Kao što je očekivano, vrijednost distorzije $(E)$ pokazuje da opća afilaktička projekcija ima najmanju distorziju. Ortogonalna je varijanta malo lošija, no estetska vrijednost ortogonalne kartografske mreže to kompenzira. Treba li karta zadovoljiti uvjet ekvivalentnosti, može se upotrijebiti ekvivalentna varijanta sa svojom još uvijek povoljnom distorzijom u usporedbi s ortogonalnom. Ekvivalentno se preslikavanje preporuča zbog distorzija koje su vidljive samo na područjima udaljenima od srednjeg meridijana.

Ekvidistantna projekcija pokazuje najlošije karakteristike distorzije. Ta varijanta ne može prikazati velika područja (vidi međuprostor na slici), njezina je vrijednost distorzije velika pa se upotreba te varijante ne preporučuje. Projekcija značajno reducira površine gotovo svugdje na karti.

Te projekcije (uz iznimku ortogonalne) akumuliraju većinu distorzija pri manjim geografskim širinama, daleko od srednjeg meridijana. Kad se izrađuje karta u tim projekcijama, ti se dijelovi mogu izostaviti. To može također biti razlogom da je ortogonalna varijanta superiorna ekvivalentnoj za Sjeverni Atlantski ocean. To područje sadrži značajan dio blizu ekvatora i udaljeno od srednjeg meridijana (Gvinejski zaljev) gdje se ekvivalentno preslikavanje pokazuje lošim. S druge strane, južni kontinenti leže blizu srednjeg meridijana, pa ekvivalentna projekcija može nadigrati ortogonalno preslikavanje (vidi vrijednosti distorzija u tablicama 1 i 2).

\section{Kratki pregled}

Poliazimutne su projekcije dobar izbor ako je područje preslikavanja veliko, leži na većim geografskim širinama i asimetrično je $u$ odnosu na jedan od polova. Njihova je prednost prikaz antimeridijana bez prekida, vrlo prilagodljive karakteristike distorzije i slike paralela koje dobro izgledaju. Posljednja navedena prednost vidljiva je samo u uspravnom aspektu projekcije. Njihovi nedostatci očituju se $u$ formulama koje su obično mnogo kompliciranije od njihovih polikonusnih analogona. Pri određivanju jednadžbi ekvivalentne poliazimutne projekcije susrećemo se $s$ implicitno zadanom funkcijom.

Poliazimutne projekcije mogu biti ekvivalentne, ortogonalne ili ekvidistantne uzduž paralela. Pored toga, one ne mogu biti konformne, osim ako se kosa stereografska projekcija ne smatra poliazimutnom. Autor prije svega preporuča ortogonalnu varijantu zbog njezinih povoljnih distorzija i estetski privlačnog izgleda ortogonalne kartografske mreže. 
why the orthogonal variant is superior to the equal-area one for the Northern Atlantic Ocean. This study area has considerable parts near the Equator and far from the midmeridian (the Gulf of Guinea) where the equivalent mapping performs poor. On the other hand, the southern continents lie near the mid-meridian where the equalarea projection could outperform the orthogonal mapping. See the distortion values in Tables 1 and 2 .

\section{Summary}

Polyazimuthal map projections are a good choice if the area of interest is large, lies on high latitudes, but is asymmetric to one of the poles. Their advantages are the uninterrupted representation of the anti-meridian, highly adaptable distortion characteristics, and good-looking circular parallels. The last advantage is only observable in the normal aspect. Their disadvantage is that their formulae are usually more complicated than that of their polyconic counterparts. An implicit function was necessary to determine the equations of the equal-area projection.

Polyazimuthal projections may be equal-area, orthogonal, or equidistant in parallels. Nevertheless, they cannot be conformal unless the oblique stereographic projection was considered polyazimuthal. The author recommends the orthogonal variant above all due to favourable distortions and its aesthetically pleasing orthogonal graticule network.

\section{References / Literatura}

Adams OS (1934) General theory of polyconic projections. Government Printing Office, Washington DC

[Bajeva] Баева ЕЮ(1987) Критерии оценки достоинства картографических проекций, исползуемых для составления карт Мира [Criteria to estimate the quality of map projections used for the composition of world maps]. Геодезия и аэрофотосъёмка по. 3, p 109-112. In Russian

Bugajevskij LM, Snyder JP (1995) Map Projections - A Reference Manual. CRC Press, London, doi: 10.1201/b16431

Canters F (2002) Small-Scale Map Projection Design. CRC Press, London, doi: 10.1201/b12656

Čebišev (Chebyshev) PL (1856) Sur la construction des cartes géographiques. Bulletin de la classe physiq.-math de l'Acad. des Sciences vol. 14, p 257-261

Frančula N (1971): Die vorteilhaftesten Abbildungen in der Atlaskartographie [The most advantageous mappings in the atlas cartography]. PhD Thesis. Reinische Friedrich Wilhelms Universität, Hohe Landwirtschaftliche Fakultät. In German.

Frančula N, Lapaine M (2018) 0 pomoćnim plohama i aspektu projekcije [Auxiliary Surfaces and Aspect of Projection] Kartografija i Geoinformacije, vol. 17, no. 29, p 84-89. In Croatian and English. https://hrcak.srce.hr/203641

Fuller B (1975) Everything I Know, Session 6, Part 6 (Oral presentation). Transcript available: https://www.bfi.org/aboutfuller/resources/everything-i-know/session-6 Accessed 30 May 2019

Györffy J (2002) Rectangular pseudopolyconic projection for geographical maps. In: Klinghammer I (ed) Studia Cartologica 12. ELTE Eötvös Kiadó, Budapest http://lazarus.elte.hu/hun/digkonyv/sc/sc12/01gyj.pdf

Kaczmarczyk G (n. d.) Downhill Simplex Method for Many ( 20) Dimensions. Available at http://paula.univ.gda.pl/ dokgrk/simplex.html Accessed 16 March 2002

Kerkovits K (2017) Vorteilhafteste flächentreue Kegelentwürfe für unregelmäßig begrenzte Gebiete [Optimizing equal-area conic and pseudo-conic projections for irregular areas]. Kartographische Nachrichten vol. 67, no. 3, p 122-128. In German with English abstract

Kerkovits K (2019) Comparing finite and infinitesimal map distortion measures. International Journal of Cartography vol. 5, no. 1, p $3-22$

Kerkovits K (in press): Quadrature Rules to Calculate Distortions of Map Projections. Under review in: The Cartographic Journal

Lapaine M, Frančula N (2016) Map projection aspects. International Journal of Cartography vol. 2 no. 1, p 38-58, doi: $10.1080 / 23729333.2016 .1184554$

Saalfeld A (1997) New Map Projection Paradigms. In: Auto-Carto XIII Proceedings of the Annual Convention and Exposition Technical Papers, Seattle, USA, 7-10 April 1997. p 347-356

[Serapinas] Серапинас ъъ (2005) Математическая картография [Mathematical Cartography]. Academia, Moscow. In Russian.

Snyder JP (1987) Map projections - A working manual. US Government Printing Office, Washington. http://pubs.er.usgs.gov/publication/pp1395

Tissot A (1878) Mémoire sur la représentation des surfaces et les projections des cartes géographiques. Nouvelles annales de mathématiques, journal des candidats aux écoles polytechnique et normale vol. 17, p 145-163

[Tolstova] Толстова ТИ (1981) Обобщенные поликонические проекции [Generalized polyconic projections]. Доклады Академии наук СCCP, vol. 259, no. 1, p 61-65 http://mi.mathnet.ru/dan44566 In Russian.

Wray T (1974) The Seven Aspects of a General Map Projection. Cartographica: The International Journal for Geographic Information and Geovisualization vol. 11, no. 2, p 1-72. doi: 10.3138/E382-8522-4783-28K5 